\title{
Plan de Acción a Partir de la Percepción en Estudiantes de la Universidad Politécnica de Sinaloa ante el Reciclaje de Residuos Sólidos y la Educación Ambiental
}

\author{
Eugenia Olaguez-Torres ${ }^{(1)}$, Piero Espino-Román ${ }^{(2)}$, Karel Acosta-Pérez ${ }^{(3)}$ y Alberto Méndez-Barceló(3) \\ (1) Universidad Politécnica de Sinaloa, Col. Genaro Estrada, Mazatlán, Sinaloa-México, Investigación y Posgrado (e- \\ mail: jolaguez@upsin.edu.mx) \\ (2) Universidad Politécnica de Sinaloa, Col. Genaro Estrada, Mazatlán, Sinaloa-México, Ingeniería Mecatrónica (e-mail: \\ pespino@upsin.edu.mx \\ (3) Universidad de Las Tunas, Facultad de Ciencias Agrícolas. Avenida Carlos J. Finlay, Las Tunas, Cuba. \\ (e-mail: karel0978@gmail.com; mendezbarcelo@gmail.com)
}

Recibido Sep. 11, 2018; Aceptado Dic. 4, 2018; Versión final Ene. 16, 2019, Publicado Jun. 2019

\begin{abstract}
Resumen
El propósito de este artículo es establecer un plan de acción para minimizar los desperdicios sólidos generados en la Universidad Politécnica de Sinaloa, México, y conocer la actitud de los estudiantes ante el reciclaje. Se utilizó una metodología de carácter descriptivo con un diseño no experimental. El instrumento utilizado consistió en un cuestionario compuesto de 14 preguntas, organizadas en dos grupos. El primer grupo busca identificar la actitud que tienen los estudiantes hacia el reciclaje y el segundo grupo identificar la concepción sobre educación ambiental. Este cuestionario fue aplicado a estudiantes de las carreras de ingeniería mecatrónica, en biotecnología, en energía, en logística y transporte y licenciatura en terapia física. El análisis permitió establecer la bases para iniciar con el proyecto de reciclaje llamado upsin-recicla, y se generaron nuevos proyectos y acciones enfocados al desarrollo tecnológico sustentables. Se concluye que este tipo de estrategias permite involucrar a la comunidad universitaria a desarrollar proyectos y fomentar una actitud favorable en beneficio del medio ambiente.
\end{abstract}

\section{Plan of Action from the Perception in Students of the Polytechnic University of Sinaloa Before the Recycling of Solid Waste and Environmental Education}

\begin{abstract}
The purpose of this article is to establish a plan of action to minimize the solid waste generated in the Polytechnic University of Sinaloa, México, and to know the attitude of students to recycling. A descriptive methodology with a non-experimental design was employed. The instrument used consisted of a questionnaire that included 14 items organized in two groups. The first group was oriented to identify the attitude of students toward recycling while the second group was aimed to identify the concept of environmental education. This questionnaire was applied to students of the careers of engineering in the areas of mechatronic, biotechnology, energy, logistic and transport, plus a group of physical therapy. The analysis allowed establishing the bases to start with the recycling project called upsin-recicla, and new projects and actions focused on sustainable technological development were developed. It is concluded that this type of strategies allows the university community to develop projects and foster a favorable attitude towards the environment.
\end{abstract}

Keywords: environmental education; sustainable development; solid waste 


\section{INTRODUCCIÓN}

Desde hace cuatro décadas el interés mundial por el medio ambiente se ha intensificado, y ha generado que diversos sectores de la sociedad se hayan preocupado por plantear acciones y programas relacionados con temas ambientales. Fenómenos como el cambio climático, impulsados sobre todo por la especie humana en el medioambiente, han dado lugar a una preocupación mundial creciente sobre el futuro y la viabilidad del planeta (Álvarez et al., 2017). Argumento que ha ocupado un lugar en la agenda global desde el año 1980, con la publicación de la Unión Internacional para la Preservación de la Naturaleza de la Estrategia de Preservación del Mundo (IUCN 1980), debido que las actividades humanas han alcanzado una proporción planetaria y llevan a la Tierra a un estado sin precedente (Steffen et al., 2004). De acuerdo con el Programa de las Naciones Unidas para el Medio Ambiente (PNUMA), dichas problemáticas son desatadas principalmente por el crecimiento demográfico humano y el desarrollo económico (PNUMA, 2012). Es así, que a partir de 1987 se empezó a socializar a nivel mundial el término desarrollo sustentable, cuyo origen se remonta a la publicación del informe Brundtland, fruto de los trabajos de la Comisión Mundial Ambiental, creada en la Asamblea de las Naciones Unidas en 1983 y lo define como: las necesidades de la generación presente, sin comprometer la capacidad de las generaciones futuras, para satisfacer sus propias necesidades. Recientemente el concepto de desarrollo sustentable ha incorporado aspectos sociales, económicos, políticos, religiosos a la temática del cuidado del medio ambiente (Pacione, 2007 y X. Yao et. al., 2009).

Fue hasta la cumbre de Río de Janeiro en 1992 que se popularizó el término de desarrollo sustentable y surgió un mayor interés internacional por los múltiples problemas ambientales; durante la cumbre se establece el plan denominado "Agenda 21", en donde dedica su capítulo 36 a la "educación, capacitación y toma de conciencia", y señala las que deberían ser prioridades de actuación en el marco de la educación ambiental, que consiste en integrar los proyectos de sustentabilidad en los centros educativos (Burgos et al., 2012). La "Agenda 21" establece las áreas de intervención de las universidades, tales como: ciencia e investigación, tecnologías, enseñanza e interacción con la sociedad civil; para fortalecer el instrumento de estrategias y acciones en temas ambientales (Curiel y Garibay, 2010). Las metas de desarrollo sustentable, es también fomentar y desarrollar nuevas aproximaciones innovadoras a la educación y la capacitación (Polis, 2005). Para hacer frente a esta problemática se ha planteado que la educación desempeña un papel importante para enfrentar a esta crisis ambiental. En el caso de México la discusión sobre la educación y gestión ambiental en el ámbito de la educación superior mexicana, tiene sus inicios desde el año de 1985 con la instalación de la Red de Formación Ambiental en Querétaro, uno de los objetivos es "contribuir a la formación y conformación de cuadros técnicos de investigadores, docentes y tomadores de decisiones, con base en la potencialidad de la oferta y demanda existente en cada región". Durante el marco del decenio de la Educación para el desarrollo Sustentable convocado por la UNESCO se presentó en México la Estrategia Nacional de Educación ambiental, un documento elaborado colectivamente que propone principios y líneas de acción presentes y futuras en materia de educación ambiental para la sustentabilidad (SEMARNAT 2010). La asociación nacional de universidades e instituciones de educación superior (ANUIES) elaboro la primer plan de acción para el desarrollo sustentable en las instituciones de educación superior (IES) en México, se establece que ninguna de las áreas del conocimiento se encuentran al margen de la problemática ambiental, además se considera que deberán ser capaces de responder a los retos de sustentabilidad con el fin de determinar las acciones educativas relacionadas con el medio ambiente y el desarrollo sustentable (ANUIES, 2009).

Lo siguiente que se realizó en México y que incluyó la participación de las IES, fue lo promovido por el consorcio mexicano de programas ambientales universitarios para el desarrollo sustentable (COMPLEXUS) a través de una serie de talleres y programas para la toma de conciencia sobre educación ambiental, algunos ejemplos de programas ambientales universitarios que participan en COMPLEXUS: Programa Ambiental Universitario de la Universidad Autónoma de Baja California, Agenda Universitaria Ambiental "AUA-UAdeC" de la Universidad Autónoma de Coahuila, Centro Universitario de Gestión Ambiental de la Universidad de Colima, Programa de Protección al Ambiente (PPA) de la Universidad Autónoma del Estado de México, Agenda Ambiental de la Universidad Autónoma de San Luis Potosí, Programa Universidad Sustentable de la Universidad de Guadalajara, Dirección de Medio Ambiente y Sustentabilidad de la Universidad de Guanajuato, Programa de Medio Ambiente de la Universidad Iberoamericana - Ciudad de México, Programa Interdisciplinario en Medio Ambiente (PIMA) de la Universidad Iberoamericana - Puebla, Programa de Ecología y Medio Ambiente (ECOULSA) de la Universidad La Salle, Sistema de Gestión Ambiental (SMA) y Educación para la Sustentabilidad (EPS) de la Universidad Tecnológica de León, Programa de Gestión Ambiental Universitario (PROGAU) de la Universidad Autónoma del Estado de Morelos, Plan Maestro para la Sustentabilidad de la Universidad Veracruzana, Plan Ambiental Institucional (PAI) de la Universidad Michoacana de San Nicolás de Hidalgo, Programa Universitario por el Ambiente (ProUPA) de la Universidad Politécnica de Aguascalientes, Programa Ambiental Universitario Chapingo (PAUCh) de la Universidad Autónoma de Chapingo y el Programa Institucional Ambiental de Desarrollo Sustentable (PIADES) de la Universidad Tecnológica del Suroeste de Guanajuato. (COMPLEXUS 2016). 
A partir de este precedente las universidades tendrán que desarrollar y fomentar programas, que contribuyan a la toma de conciencia colectiva sobre educación ambiental. Las instituciones de educación superior deben construir sus propios modelos de "universidad sustentable" (Espinosa et al., 2013). Es de resaltar que por las características de las universidades como formadoras de profesionales a desempeñarse en diversas áreas del conocimiento, es preciso atender los requerimientos de la sociedad con relación a los temas de ambiente (Nieto y Bentti, 2010), por tal motivo la educación ambiental tendrá como propósito que las personas adquieran conciencia de su entorno y puedan realizar cambios a su alcance, desde sus esquemas de valores, conductas y estilos de vida, así como ampliar sus conocimientos para impulsar los procesos de prevención y resolución de los problemas ambientales presentes y futuros (Olaguez y Espino 2013). La educación ambiental tiene como objetivo proporcionar un cambio en los valores y formas de vida que responda al doble reto (ecológico y social) que tienen planteadas nuestras sociedades (Novo y Bautista, 2012). En el campo de la educación ambiental las relaciones entre la preocupación por el ambiente, la sensibilidad para tratar el tema, conocimiento, acción y participación ante la perspectiva ambiental han sido temas de mucha investigación y debate (Esa, 2009).

Es evidente que la educación ambiental hoy en día, es un reto en la actualidad que no sólo le concierne a la educación y sus diferentes niveles, es un compromiso que demanda a hacerse de manera conjunta y con sentido de la responsabilidad que trae implícita y explicita. En tanto las instituciones de educación superior se considera que deberán desarrollar procesos de formación integral en las diversas áreas del conocimiento y a su vez incorporar la perspectiva ambiental en los programas de licenciatura, para la "detección, prevención y manejo profesional de los problemas del medio ambiente". Diversas investigaciones establecen un plan de trabajo que integra la dimensión ambiental, social y económica en las instituciones educativas (Glassey y Haile, 2012, Holmberg et al., 2012). En este sentido Varela et al. (2014) señala que la integración de la educación ambiental debe fomentar la participación de los estudiantes y docentes con el desarrollo de proyectos medioambientales. La educación ambiental, implica un proceso de carácter educativo, que además establezca esquemas cognitivos respecto a valores y actitudes, que se manifiesten en comportamientos en favor del cuidado y protección del medio ambiente (Vargas et al., 2011).

Es necesario establecer un diseño estratégico en las universidades para fomentar la educación ambiental, para ello, se debe iniciar por cuestionar qué se entiende por actitudes y comportamientos ambientales. Holahan (1991) lo define como los sentimientos favorables o desfavorables que se tienen hacia alguna característica del medio o hacia un problema con él. Para Taylor y Todd (1995) es un determinante directo de la predisposición hacia acciones a favor del medio. Es decir, las actitudes ambientales son las opiniones que se tienen acerca de proteger el ambiente y conservar los recursos (López y Quiroga, 2006). Son numerosas las investigaciones enfocadas a identificar los factores que determinan las actitudes hacia el medio ambiente (Amérigo et al. 1995, Cottrell, 2003, Guérin, 2001). Otros estudios han recurrido a la percepción de los estudiantes para obtener información relacionada con la temática ambiental para explicar, describir y predecir la actitud y valores ambientales, de esta manera determinar el comportamiento del estudiante cuando se encuentra ante un problema medioambiental (Sosa et al. 2008, Ercan, 2011, Karatekin, 2017, Espino et al, 2015, Olaguez et al. 2017).

Resulta importante y urgente promover proyectos destinados al manejo de los residuos sólidos; se estima que el promedio nacional per capital de generación de residuos sólidos es de 917 gr/hab/día en México (Sedesol, 2005), al año se producen 100,000 toneladas de envases de Polietilentereftalato mejor conocido como PET, y apenas se recicla un $20 \%$, se calcula que cada habitante desecha 6.5 kilogramos de PET al año, lo que significa que se desperdician 195 botellas de plástico, según fuentes de Asociación para Promover el Reciclaje del PET (Aprepet, 2017). La practica actual en la Universidad Politécnica de Sinaloa, México en el manejo de residuos sólidos se realiza sin segregar el tipo de residuos ya sea orgánico, papel y cartón, vidrio, plástico y material de laboratorio, no se cuenta con un registro de los residuos sólidos generados, ni del consumo de botellas de plástico dentro de la institución, una vez realizada la recolección de los residuos son entregados a los servicios municipales de recolección de basura los cuales son depositados en los rellenos sanitarios. Por lo que se hace necesario promover una actividad dentro de la universidad que ayude a mitigar el problema de la basura, al mimo tiempo general un beneficio económico para la universidad. De acuerdo con el estudio de la Secretaría de Desarrollo Social (Sedesol, 2005), se estima que los materiales recuperados para su venta (principalmente: cartón, papel, aluminio, vidrio, PET), representan del $8 \%$ al $12 \%$ del total generado. El aprovechamiento de los envases de PET, se ha convertido en una actividad rentable, fundamentalmente por las acciones encaminadas a fortalecer el acopio del material para su reprocesamiento en nuevos productos.

Con base en estas consideraciones, la presente investigación tiene el propósito de generar y aplicar estrategias constructivistas a partir de la percepción hacia el reciclaje en estudiantes de la Universidad Politécnica de Sinaloa, México, con el fin de adoptar nuevas prácticas a la conservación del medio, dado que la universidad como cualquier organización genera impacto ambiental. La Universidad deberá estar 
comprometida para formar profesionales gestores de la responsabilidad, y fomento a la cultura del reciclaje y el cuidado del medio ambiente. Para ello se deberá diseñar y poner en marcha políticas, prácticas y programas en la materia, que posibiliten dar respuestas a los problemas derivados de la actividad universitaria.

Con el precedente previamente discutido, este estudio tiene el propósito de diseñar un plan de acción que ayude a minimizar los desperdicios sólidos generados en la Universidad Politécnica de Sinaloa, México; y cumplir con el objetivo del plan institucional de la universidad, referente al cuidado del medio ambiente, y que la comunidad universitaria adquieran valores, habilidades y actitudes favorables hacia el bienestar. Para ello fue necesario conocer en primera instancia la opinión de los estudiantes acerca de la actitud que tienen hacia el reciclaje y sobre de la educación ambiental. En segundo término, fue la elaboración de la propuesta de acuerdo con la percepción interpretada de los resultados del instrumento que se aplicó a cerca del reciclaje de residuos sólidos y la educación ambiental, con el apoyo de la Organización Civil Separado no es Basura A.C. En tercer lugar la realización de conferencias con temática del medio ambiente para informar a la comunidad universitaria sobre conciencia ambiental.

\section{METODOLOGÍA}

El instrumento utilizado consistió en un cuestionario compuesto de 14 preguntas y afirmaciones. Las variables a medir fueron organizadas en dos grupos; en el primer bloque de ítems de la pregunta 1 a la 7 grupo se valoró la percepción que tienen los estudiantes hacia el reciclaje, uso del PET, medios para su gestión y tratamiento; en el segundo grupo se analizó la concepción sobre educación ambiental, posibles acciones y estrategias adecuadas para lograr su efectividad.

El estudio se limitó únicamente a la Universidad Politécnica de Sinaloa, México; la población se integró por 603 estudiantes de primer cuatrimestre de cinco programas académicos (ver figura 1). Se realizó un análisis descriptivo consistente en el empleo de medidas de tendencia central y de frecuencias para clasificar los datos acerca del tema de trabajo y determinar el alcance de los objetivos planteados al inicio de este proceso. El estudio se clasificó como un diseño no experimental, transversal y descriptivo. Se calculó un tamaño muestra de 235 estudiantes (66\% mujeres y el $34 \%$ hombres), teniendo un margen de error máximo permitido, estimado en $5 \%$ y con un nivel de confianza del 0.95 de probabilidad de que los resultados obtenidos fueran válidos. El tamaño de la muestra para la población finita y conocida se determinó con la siguiente ecuación (1):

$$
n=\frac{Z^{2}[N(p)(q)]}{e^{2}(N-1)+Z^{2}(p)(q)}
$$

\section{Dónde:}

$\mathrm{n}$ : tamaño de la muestra requerida.

Z: Valor estándar de Z=1.96 para un nivel de confiabilidad del $95 \%$.

$\mathrm{N}$ : Tamaño de la población (603 estudiantes).

$\mathrm{p}$ : Variabilidad positiva (probabilidad de valor esperado $=0.5$ )

q: Variabilidad negativa (probabilidad de valor no esperado $=0.5$ )

e: Error máximo permitido asumido (5\%)

Para la estimación de la confiabilidad del instrumento, se aplicó la medida de consistencia interna denominada alfa de Cronbach ( $\alpha$ ). El instrumento presentó un coeficiente de 0.79 , lo que representó una confiabilidad aceptable para el instrumento; toda estadística manejada se obtuvo mediante la utilización del programa estadístico EXCEL 2007.

La distribución de los estudiantes seleccionados quedó conformada de la siguiente manera 14\% estudiantes de la carrerea de ingeniería mecatrónica, 33\% estudiantes de ingeniería en biotecnología, $6 \%$ de ingeniería en energía, $33 \%$ de ingeniería en logística y transporte, $14 \%$ licenciatura en terapia física, todos del primer cuatrimestre de las carreras citadas (ver figura 1 ). 


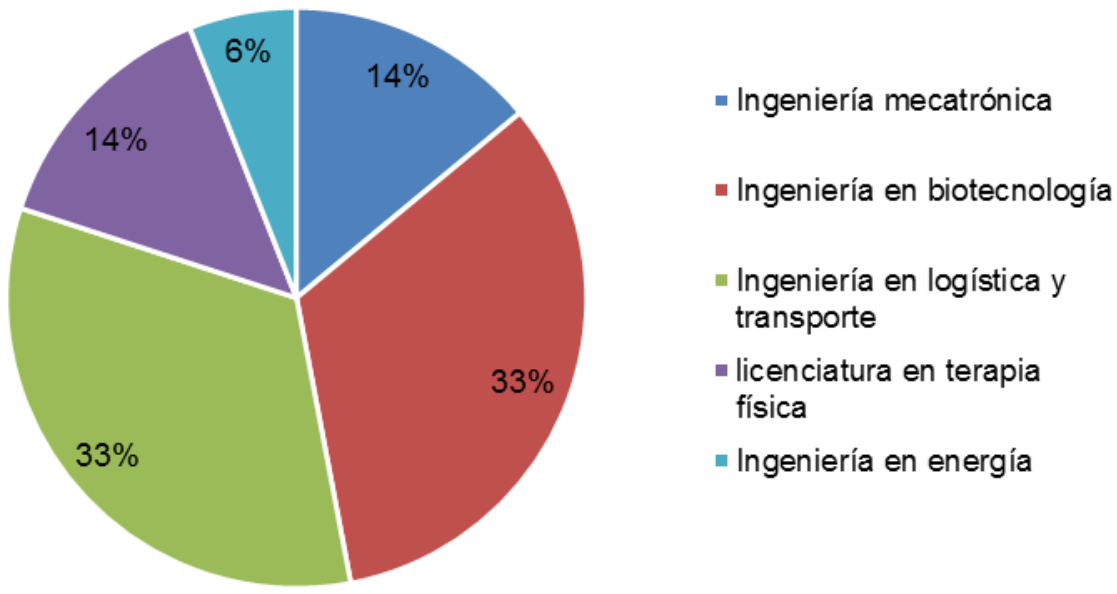

Fig. 1: Total de estudiantes encuestados

\section{RESULTADOS Y DISCUSION}

El bloque de preguntas destinadas a conocer la percepción sobre el concepto de reciclaje fueron un grupo de preguntas dicotómicas dinámicas y concretas, en donde se les cuestiono a los estudiantes sobre el concepto PET (polietilentereftalato)? acerca de las $3 R$ del reciclaje, se les pregunto a los estudiantes en un ítems exprofeso ¿Cuántas botellas de PET consumen dentro de la universidad?, la respuesta con mayor frecuencias fue de 1 a 2 "botellas diarias" con el $68 \%$, seguida con el $23 \%$ señalaron "no consumen botellas", las respuestas con menor frecuencia "3 a 4 botellas diarias" con el $8 \%$ y por ultimo con el $1 \%$ "más de 5 botellas diarias", se considera necesario conocer si los estudiantes conocen si existe centros de reciclaje en la universidad y la consideración sobre la importancia del reciclaje del PET dentro de la universidad, en medida de tener una primera aproximación acerca del involucramiento y su participación en el reciclaje del PET, ver figura 2.

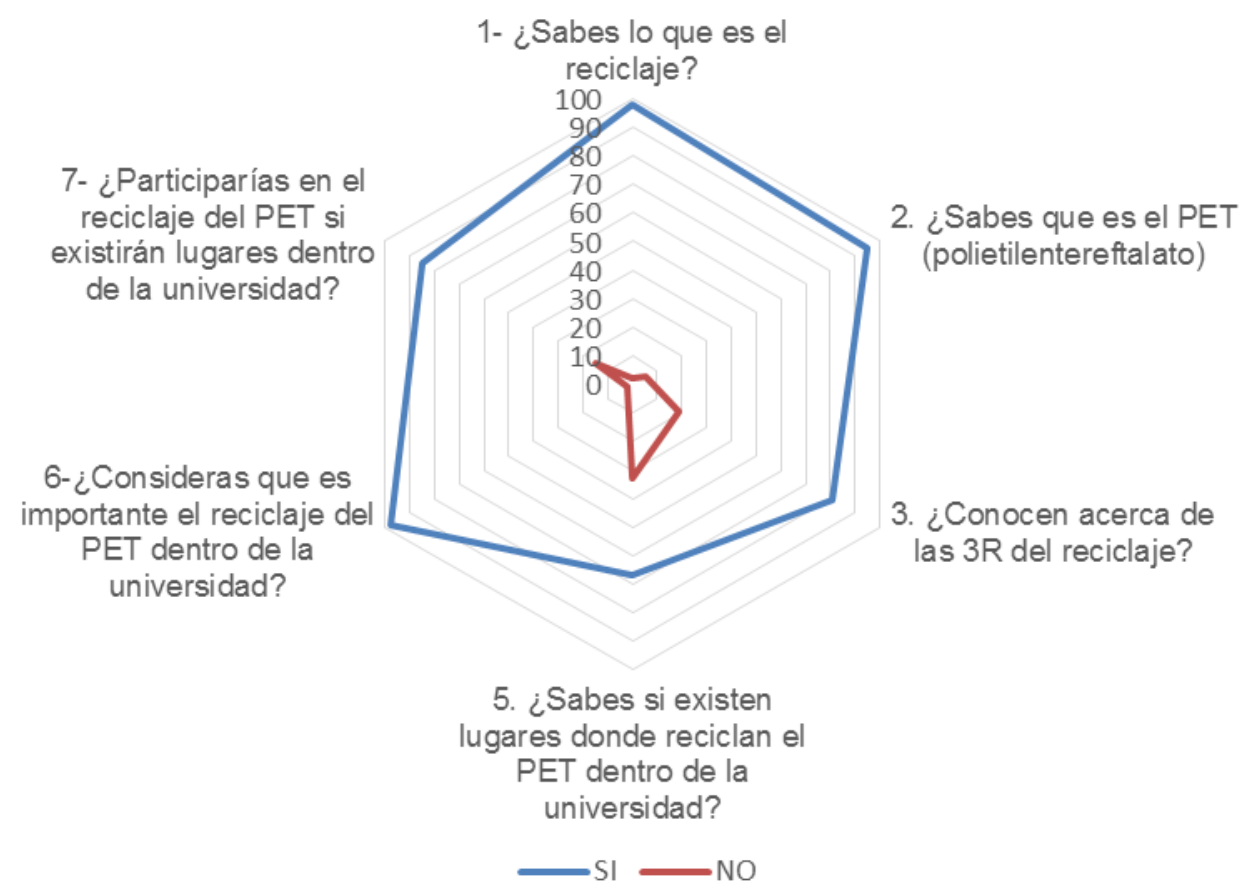

Fig. 2: Percepción de los estudiantes hacia el reciclaje.

Los resultados correspondientes a esta variable, mostraron actitudes favorables en reconocer en qué consiste el programa de reciclaje y de tener una alta disponibilidad en participar en programas que fomenten el reciclado de polietilentereftalato (PET) dentro de la universidad además que posee información sobre la ubicación de las estaciones de acopio de material reciclado en la universidad, pero a pesar de ello aún existe una apatía en realizar la separación de los residuos sólidos. Diversos estudios han descrito las 
problemática que perciben las individuos al momento de separar los residuos señalado como causa principal la limitación de espacio (Gamba y Oskamp, 1994) y la lejanía de los contenedores (Valle et al. 2004) y por otra parte, se debe ya que algunos individuos pueden evidenciar niveles elevados de preocupación ambiental y manifestar actitudes favorables hacia determinadas conductas proambientales, éstas no son suficientes para propiciar su comportamiento ecológico (Berenguer y Corraliza, 2000). Para García et al. (2011) la sociedad se declara muy sensibilizada, pero sus actuaciones no son coherentes con una participación activa en la solución de los problemas. Otro factor que influye es el nivel económico como se menciona Gallardo et al. (2013) no necesariamente tener un mayor nivel de ingreso económico significa mayor preocupación por el medio ambiente a pesar de contar con información acerca del cuidado del medio ambiente.

Por lo anterior, es de suma importancia conocer la percepción que tienen los estudiantes de la universidad con respecto de las perspectivas de la educación ambiental. En este sentido es importante conocer si los estudiantes recibieron educación ambiental a lo largo de su formación académica, el fomento de la educación ambiental en la universidad y estrategia para su implementación en actividades académicas. La encuesta incluyó algunos ítems con los que se pudo conocer la percepción de los estudiantes sobre educación ambiental. Se les pidió valoraran en una escala del 1 al 5 en la que uno (1) significó "totalmente de acuerdo" y cinco (5) "totalmente en desacuerdo" en las interpelaciones siguientes: Los casos el mayor porcentaje de respuestas se ubicaron en las escalas del cuatro a cinco, es decir, de acuerdo o totalmente de acuerdo.

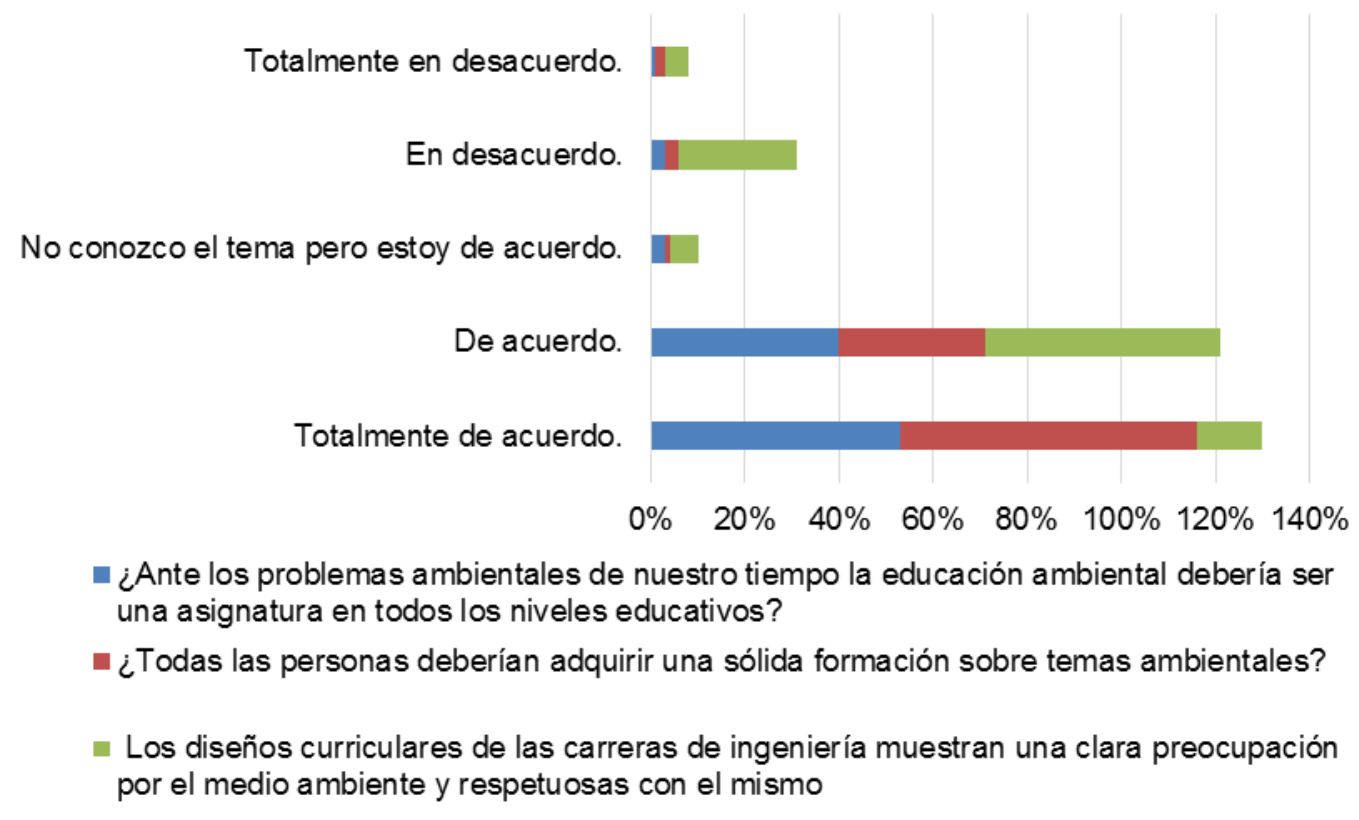

Fig. 3: Acciones de fomento de la educación ambiental

Los resultados presentados en la figura 3, refleja que los encuestados mostraron poseer un buen nivel de conocimiento acerca de educación ambiental. La educación ambiental, más allá de ser un reto es tarea individual y colectiva el contribuir en este sentido al cuidado y preservación del ambiente, integrando la conciencia, conocimiento, actitud, evaluación y participación efectiva, pues con estas acciones se considera que se fomenta la cooperatividad y el contagio crean espacios de conciencia y ejemplo así habría más posibilidad de crearse una sinergia de conciencia colectiva y holista. La utilidad de los resultados derivados de la actitud de los estudiantes hacia el reciclaje y de las acciones de fomento de la educación ambiental, permitió elaborar el plan de acción del programa de reciclaje universitario donde se involucra la participación de los estudiantes y del personal docente y administrativo.

La figura 4, muestra la utilidad de los resultados derivados de la actitud de los estudiantes hacia el reciclaje y de las acciones estratégicas propuestas para el fomento de programas que promuevan la educación ambiental en la Universidad; permitió elaborar el plan de acción del programa de reciclaje universitario donde se involucra la participación de los estudiantes y del personal docente y administrativo. 


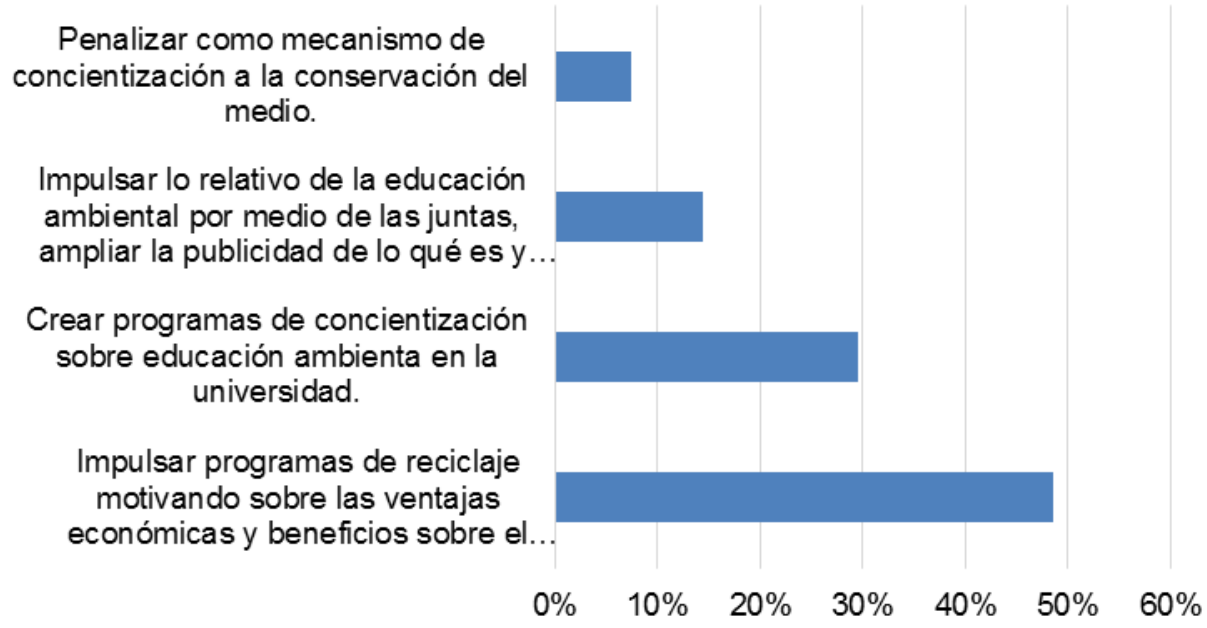

Fig. 4: Acciones estratégicas propuestas a realizarse en programas de educación ambiental en la Universidad Politécnica de Sinaloa

\section{PLAN DE ACCIÓN A PARTIR DE LA PERCEPCIÓN EN ESTUDIANTES}

La propuesta consiste en la aplicación de acciones que promuevan la cultura del reciclaje y el fomento de la educación ambiental en la universidad. Surge entonces el proyecto de educación ambiental upsin-recicla cuyo objetivo es establecer un proyecto de reciclaje, encaminado a ejercer un manejo responsable e integral de los residuos sólidos que se generan en la institución a través de un programa activo educación ambiental. Con este proyecto se impulsa uno de los objetivos contemplados en el plan de desarrollo institucional de la universidad el cual consiste en fomentar los valores, la responsabilidad social, el cuidado del medio ambiente, y el impulso a la generación de ideas que respondan al compromiso social. Fue necesario el apoyo de la Organización Civil Separado no es Basura A.C., una Organización de la Sociedad Civil que desde 2005 estableció en Mazatlán, Sinaloa, México un proyecto de separación de residuos sólidos domésticos para evitar que productos reciclables se conviertan en basura, a la vez que promueve la responsabilidad ambiental ciudadana a través de proyectos escolares y de la instalación y manejo de un centro de acopio de residuos sólidos domésticos. A través de esta asociación, se organizaron una serie de conferencias relacionadas con el cuidado del medio ambiente, la separación y tratado de desperdicios sólidos.

Se implementó un programa que incluye una serie de charlas y conferencias dirigidas a la comunidad universitaria, entre las cuales se abordaron las temáticas: del cuidado del medio ambiente, y el separado de residuos. "Por nuestro futuro común, ahora que aún es tiempo", "cambio climático", "acciones para prevenir y evitar la contaminación en ríos, playas y zonas protegidas", así como otras actividades integradoras en el marco del día internacional de la tierra y el día mundial del medio ambiente. Con las conferencias impartidas se observó que los estudiantes adquirieran, conciencia e interés para participar en proyectos de prevención y solución de problemas ambientales dentro de la universidad. Posterior al programa de charlas y conferencias, y derivado de los resultados de los cuestionarios aplicados, se realizaron tareas prácticas para la conservación del entorno de la universidad que consistió en establecer estaciones de separación de residuos sólidos, ya que los residuos se entregaban sin separación al servicio de recolección de basura del municipio. En la propuesta inicial fue diseñado para ser aplicado en cuatro etapas; la primera etapa: se realizó un programa de capacitación a toda la comunidad Universitaria sobre el separado de residuos dentro de la Universidad, donde se les explicó en forma detallada y didáctica partiendo desde la recolección, tratamiento y separación de los residuos sólidos.

La segunda etapa: La integración un grupo operativo denominado upsin-recicla este grupo tenía la función de ser eje auditor y monitor de las actividades y proyectos encaminadas al cuidado y uso responsable de la separación de residuos sólidos dentro de la universidad, además de promover la toma de conciencia de la educación ambiental con el énfasis en la cultura de la regla de las tres erres, también conocida como las tres erres de la ecología o simplemente 3R (reduce, recicla y reutiliza), es una propuesta sobre hábitos de consumo, popularizada por la organización ecologista Greenpeace, que pretende desarrollar hábitos como el consumo responsable. La tercera etapa: consiste en la colocación de dos contenedores que tiene la función de iniciar con la separación de los residuos reciclables (botellas de PET y latas de aluminio) y el otro 
para la basura común, ubicados en áreas comunes, visibles y de acceso de la universidad. La figura 5 , muestra contenedores utilizados en las estaciones. La cuarta etapa consistió en: Acopio, recolección y almacenamiento de los residuos generados; se propiciaron las condiciones para acondicionar un espacio destinado como centro de acopio, la acción de separación se realiza de forma continua y permanente, a diario los residuos fueron separados y depositados en el centro de acopio en donde se someten a una segunda separación; se pesan, registran y almacena hasta que son enviados a empresas encargadas de recibir material reciclado, mensualmente se realizó una publicación de la medición del impacto ambiental generado en el mes, mediante una bitácora ecológica cuya función es describir o ilustrar las acciones relacionadas sobre el impacto ecológico. En la figura 6, se muestra mediante un diagrama de flujo el manejo de los residuos.

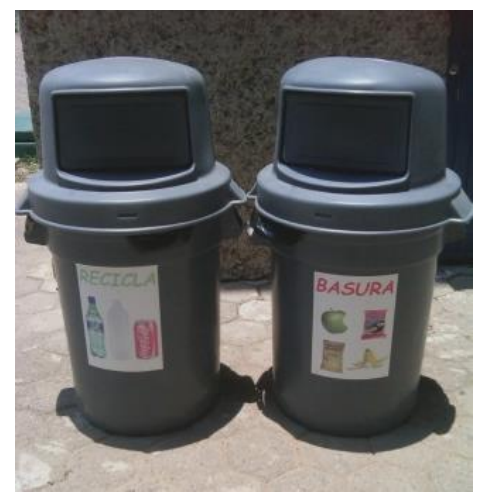

Fig. 5: Contenedores de residuos sólidos, con indicaciones graficas sobre el tipo de desperdicio que se debe depositar en cada uno de ellos.

Etapa 1

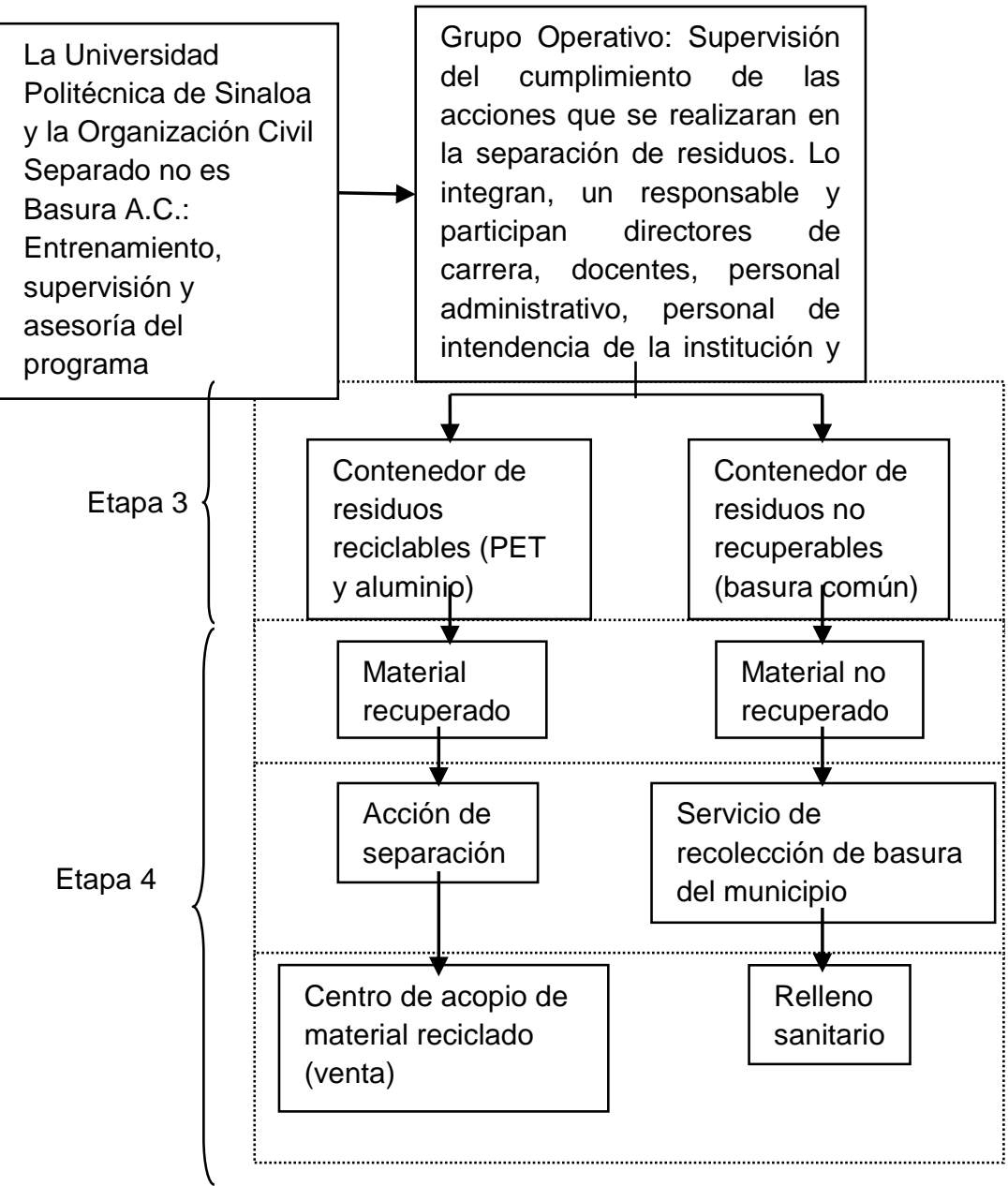

Residuos generados en la universidad

\section{Acopio}

Recolección

Destino fina

Fig. 6: Diagrama del plan de acción para el manejo de los residuos. 
A continuación se describen las funciones que realizan la Universidad Politécnica de Sinaloa, México y la Organización Civil Separado no es Basura A.C., para llevar a cabo el proyecto educación ambiental upsinrecicla: 1) La Universidad Politécnica de Sinaloa y la Organización Civil Separado no es Basura A.C., se encarga del entrenamiento, supervisión y asesoría del programa; 2) Grupo Operativo, realiza la supervisión del cumplimiento de las acciones que se realizaran en la separación de residuos. Lo integran, un responsable y participan directivos, docentes, personal administrativo, personal de intendencia de la institución y estudiantes; por ultimo 3) Acciones específicas del programa consisten en revisar estaciones, supervisión de reciclaje adecuado de los alumnos, manejo de materiales, realizar bitácora, entre otras.

En la Tabla 1, se muestran las tareas que realiza el grupo operativo upsin-recicla de la Universidad Politécnica de Sinaloa.

Tabla 1: Tareas del grupo operativo.

\begin{tabular}{|l|l|}
\hline \multicolumn{1}{|c|}{ Tarea: } & \multicolumn{1}{|c|}{ Responsable: } \\
\hline $\begin{array}{l}\text { Sensibilización sobre el problema de residuos a toda la } \\
\text { comunidad educativa. }\end{array}$ & $\begin{array}{l}\text { Organización Civil Separado no } \\
\text { es Basura A.C. }\end{array}$ \\
\hline $\begin{array}{l}\text { Planeación del operativo y su presentación a toda la } \\
\text { comunidad educativa. }\end{array}$ & $\begin{array}{l}\text { Grupo Operativo y Organización } \\
\text { Civil Separado no es Basura A.C. }\end{array}$ \\
\hline $\begin{array}{l}\text { Confirmar alumnos participantes. } \\
\text { Grupo Operativo y Organización } \\
\text { Civil Separado no es Basura A.C. }\end{array}$ \\
\hline $\begin{array}{l}\text { Elaboración de Calcomanías de identificación de residuos } \\
\text { (plástico, cartón y papel y aluminio). }\end{array}$ & $\begin{array}{l}\text { Universidad Politécnica } \\
\text { Sinaloa. }\end{array}$ \\
\hline $\begin{array}{l}\text { Definir forma de transporte de residuos al acopiador. } \\
\text { Confirmar alumnos participantes. }\end{array}$ & $\begin{array}{l}\text { Universidad Politécnica } \\
\text { Sinaloa y Grupo Operativo. }\end{array}$ \\
\hline Elaborar el impacto ambiental de los residuos acopiados. & Responsable del grupo operativo \\
\hline $\begin{array}{l}\text { Informar a la comunidad educativa sobre los resultados de los } \\
\text { acopios y el impacto ambiental (Carteles, conferencia, etc.) }\end{array}$ & Grupo Operativo. \\
\hline $\begin{array}{l}\text { Establecer el mecanismo y fechas de conteo de latas y y } \\
\text { botellas. }\end{array}$ & Grupo Operativo. \\
\hline Memoria de acopios & Grupo Operativo. \\
\hline $\begin{array}{l}\text { Reunión de evaluación con Grupo Operativo y Separado no es } \\
\text { basura para evaluar y asesorar el desempeño. }\end{array}$ & $\begin{array}{l}\text { Grupo Operativo y Organización } \\
\text { Civil Separado no es Basura A.C. }\end{array}$ \\
\hline Definir forma de transporte de residuos al acopiador & Grupo Operativo. \\
\hline $\begin{array}{l}\text { Definir fechas de las pláticas para la toma de conciencia y youán } \\
\text { conocimiento del programa Separar no es Basura. }\end{array}$ & $\begin{array}{l}\text { Universidad } \\
\text { Separado no es Basura A.C. }\end{array}$ \\
\hline
\end{tabular}

El programa upsin-recicla, ha cumplido con las etapas planteadas en el diseño del plan de acción. La etapa cuatro ha logrado durante el primer mes del programa recuperar $97 \mathrm{~kg}$ los plásticos lo cual representa un impacto ecológico y ahorro en: $100.23 \mathrm{~kg}$ de etileno, 3808.22 litros de agua, $243.083 \mathrm{Kg}$ de bióxido de carbono, $487.77 \mathrm{KW}$ de energía. En el caso de las latas de aluminio se separaron $10 \mathrm{Kg}$., lo que representa un ahorro de $40 \mathrm{Kg}$ de bauxita (mineral de que se obtiene el aluminio y representa un ahorro del $69 \mathrm{Kg}$ de bióxido de carbono y 160 KW de energía para su obtención). A partir de este plan de acción se generaron otras actividades en la Universidad en donde se promovían acciones tales como: la arborización de espacios dentro de la universidad, campañas de limpieza y mantenimiento de áreas verdes y jardines, impulsar el ahorro de energía eléctrica y el agua, participación de estudiantes en proyectos transversales generando recursos para mantener un vivero de flora regional, en cada aula se generó una acción de reciclar y separación de residuos, en cada oficina se reciclo el papel para darle doble uso y en cafetería se promovió el uso de vasos de cartón para evitar el uso de envases de plástico, así como el establecimiento de metas para que todos los programas educativos de la Institución incorporan el eje transversal del cuidado del medio ambiente.

Las anteriores acciones se incluyeron en el plan de desarrollo institucional de la Universidad Politécnica de Sinaloa, estableciéndose como objetivo principal el fomentar y consolidar una cultura de cuidado al medio ambiente en toda la comunidad UPSIN, en reconocimiento que se erige como un valor universal, que debe 
recuperarse en la formación de todo profesionista para que su práctica laboral sea con sustentabilidad (PDI 2015-2018) (Upsin, 2018). Los esfuerzos realizados por años en la Universidad Politécnica de Sinaloa en materia ambiental, se vieron reconocidos a través de la obtención del Certificado de Calidad Ambiental, otorgado por la Procuraduría Federal de Protección al Medio Ambiente. El fomento a la toma de conciencia y capacitación son factores claves para tener mejores resultados en el proceso de separación de residuos sólidos, no es fácil lograr un cambio de hábitos y actitudes hacia el medio ambiente en corto tiempo, a pesar que la comunidad universitaria mostró buena disposición a participar. Se observó que aún con las ilustraciones gráficas sobre el tipo de residuos que se debe depositar en los botes, las personas no depositaron la basura en el bote designado para tal efecto. La razón pudiera ser como lo señalan otros autores en sus investigaciones tales como Levy y Marans (2012), Weber y Duderstadt (2012), los ciudadanos se muestran interesados a participar en programas de separación de los residuos pero una falta de compromiso al momento de "aplicar" el principio. Tal (2010), señala que informales y presentarles información sobre educación ambiental contribuye en gran medida a incrementar el comportamiento de los estudiantes, pero no modifican su comportamiento proambiental. Se deberá seguir promoviendo para la toma de conciencia y capacitación, como lo señala Brito y Pasquali (2006), la consolidación de una gestión integral del manejo de la basura plantea en el inmediato y mediano plazo la motivación y ejecución de acciones que posibiliten nuevos comportamientos y actitudes ambientales, por lo que es preciso rescatar el esfuerzo, la convocatoria y el compromiso de todos los actores sociales. En otro estudio realizado por Desa et. al (2011), indica que es necesario fomentar, a través de la educación para generar conciencia sobre la gestión de residuos sólidos para poder establecer un verdadero cambio de actitud en los estudiantes.

El análisis de esta información permitirá consolidad el plan de acción en el manejo de residuos sólidos en la universidad, además de promover en la comunidad universitaria valores, actitudes e interés por el cuidado del medio ambiente, así como impulsar la participación en actividades para su protección y mejoramiento. Aún es necesario crear un medio de comunicación eficiente que proporcione información sobre los problemas ambientales y de fomentar e impulsar programas de reciclaje. Involucrar todas las áreas del conocimiento en la gestión de proyectos que promuevan el desarrollo sustentable y que además impacten en las áreas: eficiencia energética, energías renovables y tecnologías verdes.

\section{CONCLUSIONES}

De los resultados mostrados, de su análisis y de su discusión, se pueden obtener las siguientes conclusiones:

1) El resultado de las encuestas permitió establecer las estrategias para minimizar los desperdicios de los residuos sólidos y fomentar la cultura del medio a través de la separación y reciclado;

2) El plan de acción a partir de la percepción de los estudiantes busca un valor agregado para la comercialización del material reciclado generado en la universidad, además de establecerse en uno de los ejes estratégicos del plan de desarrollo institucional de la universidad;

3) Se involucró la comunidad universitaria a desarrollar proyectos que promueven la educación ambiental y fomentar una actitud favorable en beneficio del medio ambiente siendo posible a través de las asignaturas del bloque transversal de humanidades;

4) El estudio pone en evidencia la importancia de contribuir con el cuidado de medio ambiente por parte de la universidad y de asociaciones civiles. 5) Los estudiantes encuestados se perciben consientes y dispuestos a involucrarse a realizar mayores acciones que promuevan la sustentabilidad y la cultura del cuidado del medio.

\section{REFERENCIAS}

Álvarez, M.M., A. Arias, M.A. Lorenzo y F. Serrallé, Educación para la sustentabilidad: cambio global y acidificación Oceánica, doi: 10.4067/S0718-50062017000200010, Formación Universitaria, 10(2), 89-102 (2017)

Amérigo, M., A. González y J.I. Aragonés, Antropocentrismo versus egocentrismo en una muestra de estudiantes, Psicología Política, Jurídica y Ambiental, 337-344, Eudema, Salamanca, España (1995)

Anuies, Plan de acción para el desarrollo sustentable en las instituciones de educación superior, Asociación Nacional de Universidades e Instituciones de Educación Superior (2009)

Aprepet, Reciclaje: un negocio rentable, Asociación para Promover el Reciclaje del PET (2017)

Baldi, G. y E. Quiroga, Una aproximación la psicología ambiental, ISSN: 1515-4467, Fundamentos en Humanidades, 7(1), 157-68 (2006)

Berenguer, J. y J.A. Corraliza, Preocupación ambiental y comportamientos ecológicos, ISSN: 0214 - 9915, Psicothema, $12(3), 325-329(2000)$ 
Brito, E. y C. Pasquali, Comportamientos y actitudes asociados a la disposición de la basura en áreas urbanas no planificadas, ISSN: 0378-1844, Interciencia, 31(5), 338-344 (2006)

Burgos, O., J. Gutiérrez y F.J. Perales, La evaluación de la calidad en las eco-escuelas: un estudio comparado entre Chile y España, ISSN: 0378-1844, Interciencia, 37(5), 340-347 (2012)

COMPLEXUS, Consorcio Mexicano de Programas Ambientales Universitarios para el Desarrollo Sustentable (2016)

Cottrell, S.P., Influence of sociodemographic and environmental attitudes on general responsible environmental behavior among recreational, doi: 10.1177/0013916503035003003, Environment and Behavior, 35(3), 347-375 (2003)

Curiel, A. y G. Garibay, Indicadores de primera generación para medir los aportes de las universidades al desarrollo sustentable (2010)

Desa, A., N. Ba'Yah-Abdul-Kadir y F. Yusooff, A Study on the Knowledge, Attitudes, Awareness Status and Behaviour Concerning Solid Waste Management, doi:10.1016/j.sbspro.2011.05.095, Procedia Social and Behavioral Sciences 18, 643-648 (2011)

Ercan, F., Student perceptions and solutions about the matters of environment, doi: 10.1016/j.sbspro.2011.05.153, Procedia Social Behavional Sciencies, 19, 450-452 (2011)

Esa, N., Environmental knowledge, attitude and practices of student teachers, doi: 10.1080/10382040903545534, International Research in Geographical and Environmental Education, 19 (1), 39-50 (2009)

Espino, P., E. Olaguez e Y.A. Davizon, Análisis de la percepción del medio ambiente de los estudiantes de ingeniería en mecatrónica, doi: 10.4067/S0718-50062015000400006, Formación Universitaria, 8(4), $45-54$ (2015)

Espinosa, R.M., S. Turpin y otros cinco autores, La gestión ambiental en una institución de educación superior asociada a las prácticas de separación y recuperación de residuos, ISSN: 0188-4999, Revista internacional de contaminación ambiental, 29,49-57 (2013)

Gallardo, A., A. Gómez y otros tres autores, Influencia del nivel de ingresos económicos en la recogida selectiva de residuos urbanos, ISSN: 0188-4999, Revista internacional de contaminación ambiental, 29,19-24 (2013)

Gamba, R. y S. Oskamp, Factors influencing com-munity residents participation in commingled curbside recycling programs, doi: 10.1177/0013916594265001, Environment and Behavior, 26, 587-612 (1994)

García, J. y P. Navarro, ¿Qué piensan y cómo dicen que actúan los alumnos y profesores de un Centro de Educación Secundaria sobre la gestión del agua, la energía y los residuos?, Enseñanza de las ciencias, 29(1), 61 -74 (2011)

Glassey, J. y S. Haile, Sustainability in chemical engineering curriculum, doi: 10.1108/14676371211262308, International Journal of Sus-tainability in Higher Education, 13, 354-364 (2012)

Guérin, D., J. Crête y J.A. Mercier, Multilevel analysis of the determinants of recycling behavior in the European Countries, doi: 10.1006/ssre.2000.0694, Social Science Research, 30(2), 195-218 (2001)

Holahan, C.J., Psicología Ambiental: Un enfoque general, 43-44, Editorial Limusa S.A. De C.V., México (1991)

Holmberg, J., U. Lundqvist, M. Svanström y M. Arehag, The university and transformation towards sus-tainability: The strategy used at Chalmers University of Technology, doi: 10.1108/14676371211242544, International Journal of Sustainability in Higher Education, 13, 219-231 (2012)

IUCN, World Conservation Strategy: Living Resource Conservation for Sustainable Development, Union for Conservation of Nature and Natural Resources, UNEP, WWF, FAO, UNESCO (1980)

Karatekin, K., Perception of environmental problem in elementary students' mind maps, doi: 10.1016/j.sbspro.2013.09.295, Procedia Social Behavional Sciencies, 93, 868-872 (2013)

Levy, B. y R. Marans, Towards a campus culture of environmental sustainability: Recommen-dations for a large university, doi: 10.1108/14676371211262317, International Journal of Sustainability in Higher Education, 13(4), 365$377(2012)$

Nieto, Y.A. y A.S. Bentti, Proyecto para la acción: estrategia de educación ambiental a nivel universitario ante la problemática local y global, ISSN: 1316-6077, Geoenseñanza 14(2), 327-333 (2009)

Novo, M. y M.J. Bautista, Análisis de la incidencia de la educación ambiental para el desarrollo sostenible en las revistas científicas españolas, doi: 10-4438/1988-592X-RE-2012-358-176, Revista de Educación, 358, 583-597 (2012)

Olaguez, E. y P. Espino, Perspectivas de educación ambiental en las instituciones de educación superior, ISSN: 20079990, Revista Iberoamericana de las Ciencias Biológicas y Agropecuarias, 2(3) (2013)

Pacione, M., Sustainable urban development in the UK: rhetoric or reality? Geography, 92 (3), 248-265 (2008)

Pnuma, Perspectivas del medio ambiente mundial, Programa de las Naciones Unidas para el Medio Ambiente GEO 5 (2012)

Polis, Usando Ciencia, Tecnología e Innovación para el Desarrollo Sustentable, ISSN: 0717-6554, Revista de la Universidad Bolivariana de Chile, 4 (12) (2005)

Sedesol, Manual técnico sobre generación, recolección y transferencia de residuos sólidos municipales, Secretaria de Desarrollo Social (2005) 
Semarnat, Transversalidad de políticas públicas para el desarrollo sustentable, Secretaria del medio ambiente y recursos naturales (2010)

Sosa, M., J. Alcalá y otros tres autores, Percepción ambiental de estudiantes universitarios a través de variables medioambientales, Revista Latinoamericana de Recursos Naturales, 4, 178 -184 (2008)

Steffen, W., A. Sanderson y otros ocho autores, Global Change and the Earth System: A Planet under Pressure (2004)

Tal, T., Pre-Service Teachers' Reflections on Awareness and Knowledge Following Active Learning in Environmental Education, doi:10.1080/10382046.2010.519146, International Research in Geographical and Environmental Education, 19 (4), 263-276 (2010)

Taylor, S. y P. Todd, An integrated model of waste management behavior. A test of household recycling and composting intentions, doi: 10.1177/0013916595275001, Environment and Behavior, 27(5), 603-630 (1995)

Upsin, Plan de desarrollo institucional 2015-2018, Universidad Politécnica de Sinaloa (2018)

Valle, P., E. Reis, J. Menezes y E. Rebelo, Behavioral determinants of household recycling participation: the Portuguese case, doi: 10.1177/0013916503260892, Environment and Behavior, 36, 505-540 (2004)

Varela, M., U. Pérez, F.J. Álvarez y M.M. Álvarez, Desarrollo de competencias docentes a partir de metodologías participativas aplicadas a la educación ambiental, doi:10.4067/S0718-50062014000600004, Formación Universitaria, 7(6), 27-36 (2014)

Vargas, C., J. Medellín, I. Vázquez y G. Gutiérrez, Actitudes ambientales en los estudiantes de nivel superior en México, ISSN: 1909-2474, Revista Luna Azul, (33), 31-36 (2011)

Weber, L. y J. Duderstadt, Global Sustain-ability and the Responsibilities of Universities, $1^{\text {a }}$ Ed., Glion Colloquium Series, No 7, Económica, Londres, Paris y Ginebra (2012)

Yao, X., C. Watanabe y L. Ying, Institutional structure of sustainable development in brics: Focusing on ict utilization, doi: 10.1016/j.techsoc.2008.10.013, Technology in Society, 31, 9-28 (2009) 